\title{
Costs of Responding to a Measles Outbreak: Buvuma Islands, Lake Victoria, Uganda, February- May 2017
}

Joyce Nguna ( $\sim$ joynguna@musph.ac.ug )

Kementerian Kesihatan Malaysia

Joy Kusiima Bbaale

Uganda Public Health fellowship Program

Doreen Birungi

Uganda Public Health Fellowship Program

Benon Kwesiga

Uganda Public Health Fellowship Program

Daniel Kadobera

Uganda Public Health Fellowship Program

\section{Bernard Toliva Opar}

Ministry of Health

Immaculate Ampaire

Ministry of Health

Henry Luuze

Ministry of Health

Brendan Kwesiga

Management Science for Health

Wilbrod Mwanje

African Field Epidemiological Network

Alex Riolexus Ario

Ministry of Health, Public Health Fellowship Program

Research article

Keywords: Measles, Outbreak, Cost, Vaccination, Buvuma Islands, Uganda

Posted Date: August 23rd, 2019

DOI: https://doi.org/10.21203/rs.2.13457/v1 
License: (c) (i) This work is licensed under a Creative Commons Attribution 4.0 International License. Read Full License 


\section{Abstract}

Background Despite the strong prevention efforts by the Uganda Ministry of Health ( $\mathrm{MOH})$, measles outbreaks continue to occur. The $\mathrm{MOH}$ responded to a measles outbreak in the hard to reach areas of Buvuma Islands, identifying 54 case-patients, 4 of whom developed complications and were hospitalized. We estimated the provider cost of responding to this outbreak, cost of prevention, and the cost the government would have saved with effective prevention. Methods We took the Government perspective into consideration. Using an itemized form, we systematically collected data on quantities and unit costs of all the resource inputs for both direct and indirect costs at national, district, and facility levels. Medical cost referred to hospital and clinic costs for medications, supplies, utilities, transport, and personnel; nonmedical costs included those associated with person-hours spent on the outbreak investigation and control effort. Results The overall cost of investigating and controlling this outbreak was $\$ 16,259.50$ (including $\$ 5,526.30$ ) of medical costs, $\$ 10,733.20$ of non-medical costs) and the cost per capita of number of children 6mon-5years was $\$ 117.80(16,259.5 / 138$ (number of children 6 mon-5years. This is the target for measles intensified immunization following an outbreak). Conclusion and Recommendations The total cost incurred in this outbreak is four fold the amount needed to vaccinate all children in Buvuma which would have averted the outbreak. We recommend strengthening vaccination services in all corners of the country, especially hard-to-reach areas, to enable the government forego the extra cost and morbidity associated with outbreak control.

\section{Background}

Measles is a highly-transmissible acute infection caused by a paramyxovirus, and characterized by fever, conjunctivitis, generalized maculopapular skin rash, and respiratory symptoms [1]. Management of measles involves symptom management including fever, rash, conjunctivitis and coryza. Prior to the availability of measles vaccine, measles infected over $90 \%$ of children before they reached 15 years of age. These infections were estimated to cause more than two million deaths and between 15,000 and 60,000 cases of blindness annually worldwide[2,3].

In 2005 , the WHO set a goal of eradicating $90 \%$ of measles mortality by 2010 ; this was adopted globally by African countries [1]. Despite remarkable progress towards global reduction of measles morbidity and mortality, measles cases are still high in developing countries especially in Africa [4].

According to Uganda $\mathrm{MOH}$ reports, the Uganda National Expanded Program on Immunisation (UNEPI) has documented huge successes in terms of vaccination coverage since 1983 and concentrated on vaccination efforts to cover the target population. In Uganda, the measles vaccine has been given at 9 months as part of the routine immunization schedule since 1995 [5]. The government of Uganda has for the past years improved measles vaccination coverage through routine immunization, periodic intensified routine immunization (PIRI) and supplementary immunization activities. Clear evidence is also exhibited in Uganda where measles control strategy for the period 2002-06, including the measles vaccination 
campaign for children under 15 years, follow-up campaigns and rising routine immunization coverage, led to a dramatic (93\%) and $100 \%$ reduction in measles incidence and measles mortality respectively [6].

The District Health Information System (DHIS2) data for 2013, 2014 and 2015 puts national measles coverage, at $97 \%, 96 \%$ and $106 \%$ respectively. However, the current national preliminary multi-indicator cluster survey report, recorded this coverage at $80 \%$. Although administrative data shows that the measles immunisation coverage is high, Uganda has in the past several years been experiencing recurrent measles outbreaks which have been attributed to low immunisation coverage in some of the districts [6-8].

According to routine health information reports and coverage surveys, measles immunization coverage increased from 17\% in 1985 to 82\% in 1995, declined between 1996 to 1998, and then stabilized from 1999 to 2001 at approximately 61\% [8]. In 2014 and 2015 the administrative vaccination coverage for measles stood at $94 \%$ and $106 \%$ respectively[8].

Numerous studies have demonstrated the cost-effectiveness and significant net economic benefits of measles and rubella vaccination efforts [9]. In 1985, White and colleagues reported economic benefits that significantly exceeded the costs associated with using Measles Mumps Rubella (MMR) vaccine for routine vaccination in the United States of America (USA) [10].

In addition to the cost incurred due to individual measles cases, investigating and responding to outbreaks imposes an economic burden on the healthcare system and community [11].

On $14^{\text {th }}$ March 2017, the Uganda Ministry of Health $(\mathrm{MOH})$ was notified of a measles outbreak on the Islands of Lake Victoria, Buvuma District. A response team from the Ministry including the Uganda Public Health Fellowship Programme (PHFP), Uganda National Expanded Programme on Immunisation (UNEPI), and Uganda Virus Research Institute (UVRI) supported by the African Field Epidemiology Network (AFENET) and the Buvuma District Health Team conducted a thorough investigation and controlled the measles outbreak. The composition of the response team included epidemiologists, laboratory technologists and district health workers. By the end of the outbreak, a total of 54 cases were identified; 4 were hospitalized with complications.

Several studies have provided estimates of the cost of responding to measles virus infections and containing outbreaks in high income countries however, the economic burden of measles outbreaks in low and middle countries is not clear[12]. While the costs associated with vaccination are well-known, cost analyses of vaccine-preventable diseases among children below 5 years of age in Uganda are limited, particularly the costs of responding to outbreaks. Cost estimates of measles outbreaks and response activities in Uganda would provide evidence for improving national immunization policies, and demonstrating the economic benefits of measles eradication. We estimated the provider cost of responding to this outbreak, cost of prevention and the cost government would have saved with effective preventive measures including immunization and effective surveillance. 


\section{Methods}

Study site

Buvuma District, Uganda comprises a collection of 52 Islands in Lake Victoria, with no territory on the mainland, and includes $~ 55,300$ people[13] (Figure 1).. The measles vaccine coverage in Buvuma District in 2016 was $88 \%$ (UNEPI target: $90 \%$ ).

\section{Study design}

We defined a provider as public entity where services are offered for free and was incharge of coordinating the outbreak response activities. We conducted a descriptive cross-sectional study putting the government perspective into consideration and systematically collected cost data on resource inputs at national, district, and facility levels. We defined the study period as March to May 2017(when the MOH was first notified about the measles outbreak through to the date when the outbreak was declared over). Quantifying the cost of illness involved quantification of the direct medical costs and non-medical costs involved in measles treatment and care. We used a case definition to classify all identified patients as suspected or confirmed measles cases. A total of 20 people participated actively in managing the outbreak; these included; 5 District Health Team (DHT) members, 5 implementing partner representatives who financially supported the outbreak response, $6 \mathrm{MOH}$ staff and 4 drivers. The DHT were involved in patient management, health education and surveillance whereas all the partners and $\mathrm{MOH}$ staff were involved in the measles investigation. The investigation of the measles outbreak lasted 30 days.

\section{Data management and quality assurance}

\section{Data collection}

The data were pretested in a pilot study of 10 patients to ensure the feasibility of the study and its methodology as well as give an understanding on the overall cost of therapy. We recorded all the data in our costing checklist (additional file 1). The data were cleaned and any errors or omissions corrected. The data was then transferred onto Excel spreadsheets, which were only accessed by the investigators.

We then listed all activities from stakeholders that participated in the response in a chronological order. We made a list of resource inputs with their data sources and compared them to form the basis of a set of itemized sheets for the different activities. We then used an itemized form to collect all cost data from the District Health Office (DHO), health workers, Chief Administrative Officer (CAO) of Buvuma District, $\mathrm{MOH}$ (national) and partners. The required quantities of inputs and resources were based on estimates by the Uganda Clinical Guidelines (UCG), 2017 for measles management and using expert opinion (physician) (Table 1)..

Assessment of outbreak costs 
The assessed costs that included medical costs and non-medical costs are shown in Table 2 below. An activity list was made with all corresponding resource inputs. The resource inputs were valued, measured, and summed to get the total cost spent. All costs were expressed in Uganda shillings and later converted into US dollars as of May 2017 exchange rates.

\section{Medical costs}

We made a list of item of all activities we conducted during the investigation, for example resource inputs. We collected information on the number of health facility visits and number of complicated and uncomplicated measles cases from health facility in-charges (usually nurses or doctors), lay caretakers (usually family members). We compiled lists with names of all measles cases, and we searched other medical records of the same cases for any other patient details that were missing in the line list. Using phone calls, we obtained more information about the cases from their caretakers. We calculated personnel costs by multiplying a person's daily salary by the number of days spent particularly on the measles outbreak. According to the Uganda Clinical Guidelines, 2017, measles complications (otitis media, pneumonia, conjunctivitis, diarrhea, vomiting, dehydration, encephalitis and upper respiratory infection) do not require treatment but rather symptom management. We estimated the cost of treating a measles case at $\$ 6.00$ based on estimates from global eradication for measles that included Uganda[14].The estimate cost was multiplied over an average time of illness which was 4 days with a range of 5-7 days.

\section{Non-medical costs}

\section{Personnel hours}

We calculated the personnel hours lost which included both business and nonbusiness hours (weekends) allocated to the response team. We estimated the number of hours lost, converted them to days and then valued the days based on average pay per day. We estimated the salary for the response team based on the Uganda Bureau of Statistics labour Survey.

\section{Costs at the National Level-National coordination team}

We calculated personnel time of the National Task Force (NTF) by hours dedicated to surveillance, response, laboratory work, health education, and coordination team activities related to this particular outbreak at national level. Given the limited resources, this allocation of personnel time represents the loss of other productivity at work (i.e. opportunity costs). The departments largely involved were; UNEPI, PHFP, Epidemiology and Surveillance Division (ESD) of $\mathrm{MOH}$, Uganda Virus Research Institute and the Uganda Ministry of Health Emergency Operations Centre and their time estimates were obtained from personal interviews with personnel in the relevant departments. We calculated personnel costs by multiplying a person's daily salary by the number of days spent on the measles outbreak and added the allowances/perdiem for the same period. We computed costs of communication in terms of telephone 
calls made by EOC to Buvuma on a daily basis for the one week when the outbreak was first reported to $\mathrm{MOH}$.

\section{Costs at District Level}

To estimate the amount of personnel time associated with local outbreak response activities, we administered a checklist to all members of the district rapid response team that were available. We also collected local reports and consulted registries for additional information. We calculated time and costs per notified case in Kibibi village and extrapolated these estimates to all notified cases. The DHT confirmed that case finding often involved a boat ride which was time consuming and costly, so water transport costs were also included. On average, these outbreak investigation activities required 1-2 hours per case. We also costed the internal staff meetings that were held to organize district response activities at the beginning of the outbreak. The unit cost used for a fully immunized child was estimated at $\$ 33.7$ for 1 dose of measles vaccine[15].

\section{Costs of Prevention}

We estimated the cost of prevention as the total cost of vaccination for all children aged 6 months to 59 months. We abstracted secondary data from the district health office to get the number of children below 5 years in Buvuma district. We estimated the cost of immunizing a child against measles at $\$ 33.7$ from the immunization Costing report, 2015. We then multiplied the approximate number of children below 5 years on Kibibi Island with the cost of full immunization. The number of targeted children as is calculated in Immunization was computed as an approximate proportion for measles campaigns of $18.13 \%$ of the total population for Kibibi Island respectively. We then multiplied the cost of measles vaccine per child by the targeted population for prevention.

\section{Results}

\section{Costs of response}

\section{Provider costs}

In total, 54 cases of measles infection with 4 complications were reported during an outbreak on Kibibi Island, Buvuma District. The overall provider cost incurred in investigating and responding to the measles outbreak was $\$ 16,259.5$ as shown in Table 2 . The cost per capita for responding to the outbreak on Kibibi Island was $\$ 117.8(16,259.5 / 138)$. The medical costs totaled $\$ 5,526.3$ including treatment costs; $\$ 363$, utility costs; $\$ 100$ and transport and fuel costs of $\$ 5,063$.3. The non-medical costs totaled to $\$ 10,733.2$ including personnel costs of $\$ 10,436.2$ and overhead costs of $\$ 297.2$. The personnel costs included salary lost (were given salary but had not worked) during the outbreak period (\$5862) and allowances given to the response team (\$4574). Buvuma district health office needed $\$ 4642.3(33.7 * 138)$ to immunize all the children 6 months to 5 years on Kibibi Island. This outbreak posed substantial logistical 
challenges for the DHT, most importantly with the high fuel costs $(\$ 5,063.3)$ needed to navigate the islands.

\section{Cost of prevention}

The total population for Kibibi Island was 760 persons and approximate target population for vaccination was 138 . We estimated the cost of immunization at Kibibi Island at $\$ 6099.6$ including all related costs respectively.

\section{Discussion}

Our study found that the overall provider cost of investigating and responding to the measles outbreak in Buvuma Islands was $\$ 16,259.5$. Buvuma district health office needed $\$ 4642.3(33.64 * 138)$ to immunize all the children 6 months to 5 years on Kibibi Island. Therefore this depicts besides responding to the outbreak, the provider needed to spend $\$ 11,617.11(16,259.5-4642.3)$ more to control this outbreak than it would have spent to prevent it. These findings agree with a study in Uganda which noted supplementary immunisation activities as a cheaper option than medical and other costs involved in managing measles cases [16].

However a study in Burundi about a measles outbreak in Muyinga Sector, Burundi documented that conducting outbreak control or disease elimination may not be the most cost effective use of limited resources but rather appropriate therapy for the diseased cases [17].

Our findings were also higher than costs $(\$ 475)$ of laboratory tests for 360 neonates that succumbed to a meningitis infection in Tanzania[18] This could be attributed to the differences in cost of treatment regimens for measles and meningitis

Our findings are also congruent with the USA where hospital and outbreak response costs were found to be higher than preventative costs for measles [19].

Comparable to our findings, in Japan a study concluded that the policy of immunizing infants for measles soon after their one-year birthday is economically effective [20].

Our estimated hospitalization costs are much lower than findings in Italy [21], but studies conducted in the Netherlands and Canada [16] recorded lower costs of \$276 and \$254 respectively. Nonetheless the differences in outbreak control costs recorded in different countries vary with variability of currencies and standards of treatment. Even though the findings in Italy were much higher, they are in line with our findings in that the costs of response were higher than those for vaccination.

\section{Limitations}

Our findings should be interpreted in line with the following limitations. The overall cost of responding to the outbreak could have been underestimated since we did not compute boat and vehicle depreciation 
and building maintenance. In-addition, the total cost could have been underestimated since we may have missed unreported cases in the community. Failure to reach some areas could have caused an underestimation of the overall cost for responding to the outbreak.

\section{Conclusions}

Despite high vaccination coverage, measles outbreaks can occur among under-vaccinated children, at major cost to public health agencies. The total cost incurred in this outbreak would have been more than enough to vaccinate all children on Kibibi Island in Buvuma, which would have prevented the outbreak. This would be a recurring extra cost the government would have to expend if the routine vaccination is suboptimal and an outbreak occurs. Rising rates of intentional under vaccination e.g were community people don't turn up for Immunization campaigns, this can undermine the global goal onwards measles elimination. We recommend that the government should encourage people do vaccination to avert future illnesses and potentially related death instead of responding to the outbreaks. Preventive strategies including public education to optimize the use of local health resources such as immunization should be encouraged.

\section{Abbreviations}

AFENET: African Field Epidemiology Network EOC: Emergency Operations Centre AR: Attack Rate Cl: Confidence Interval DHO: District Health Office DHT: District Health Team DSFP: District Surveillance Focal Person IDSR: Integrated Disease Surveillance and Response JMS: Joint Medical Stores MOH: Uganda Ministry of Health NMCP: National Malaria Control Program PHFP:Uganda Public Health Fellowship Program SCD: Standard Case DefinitionUCG: Uganda Clinical Guidelines VHT: Village Health Team

\section{References}

1.WHO., World Health Organisation. Report on the 1st consultation of the technical Advisory Group on Measles and Rubella Control in the African Region. 2005.

2.WHO, Global measles and rubella strategic plan: 2012. World Health Organization, 2012.

3.Strebel, P.M., et al., A World Without Measles. The Journal of Infectious Diseases, 2011. 204(suppl_1): p. S1-S3.

4.Grais, R., et al., Unacceptably high mortality related to measles epidemics in Niger, Nigeria, and Chad. PLoS Med, 2007. 4(1): p. e16.

5.WHO, World Health Organization. Measles vaccines: WHO position paper.. Wkly Epidemiol Rec, 2009. 84(349-60). 
6.Mbabazi, W. B., et al., Achieving measles control: lessons from the 2002-06 measles control strategy for Uganda. Health policy and planning, 2009. 24(4): p. 261-269.

7.Nsubuga, F., et al., Factors contributing to measles transmission during an outbreak in Kamwenge District, Western Uganda, April to August 2015. BMC infectious diseases, 2018. 18(1): p. 21.

8.Ministry of Health, U., District Health Information System 2. 2002.

9.Simons, E., et al., Assessment of the 2010 global measles mortality reduction goal: results from a model of surveillance data. The Lancet, 2012. 379(9832): p. 2173-2178.

10.White, C. C., J. P. Koplan, and W. A. Orenstein, Benefits, risks and costs of immunization for measles, mumps and rubella. American journal of public health, 1985. 75(7): p. 739-744.

11.Dayan, G. H., et al., The cost of containing one case of measles: the economic impact on the public health infrastructure-lowa, 2004. Pediatrics, 2005. 116(1): p. e1-e4.

12.Goodson, J. L., et al., Research priorities for global measles and rubella control and eradication. Vaccine, 2012. 30(32): p. 4709-4716.

13.GOU., National Housing and Population Census. Government of Uganda 2014.

14.Levin, A., et al., Global eradication of measles: an epidemiologic and economic evaluation. The Journal of infectious diseases, 2011. 204(suppl_1): p. S98-S106.

15.Teresa Guthrie, C. Z., Brendan Kwesiga, Christabel Abewe, Stephen Lagony, Carl Schutte, Edmore Marinda, Kerrin Humphreys, Zipozihle Chuma Nombewu, Katlego Motlogelwa, Anthony Kinghorn, Costing and Financing Analyses of Routine Immunization in Uganda; Techinical Report Health Development for Africa 2014.

16.Carabin, $\mathrm{H}$., et al., The average cost of measles cases and adverse events following vaccination in industrialised countries. BMC Public Health, 2002. 2(1): p. 22.

17.CHEN, R. T., et al., A 'post-honeymoon period'measles outbreak in Muyinga sector, Burundi. International journal of epidemiology, 1994. 23(1): p. 185-193.

18.Vaagland, H., et al., Nosocomial outbreak of neonatal Salmonella enterica serotype Enteritidis meningitis in a rural hospital in northern Tanzania. BMC infectious diseases, 2004. 4(1): p. 35.

19.Chen, S. Y., et al., Health care-associated measles outbreak in the United States after an importation: challenges and economic impact. Journal of Infectious Diseases, 2011. 203(11): p. 1517-1525.

20.Takahashi, K., Y. Ohkusa, and J.-Y. Kim, The economic disease burden of measles in Japan and a benefit cost analysis of vaccination, a retrospective study. BMC health services research, 2011. 11(1): p. 254. 


\section{Tables}

Table 1: Inputs classified in broad categories

\begin{tabular}{|l|l|}
\hline Category & Items \\
\hline Drugs used & Vit A, Paracetamol. Zinc kid, Amoxyl, Eye ointment(tetracycline) \\
\hline $\begin{array}{l}\text { Medical } \\
\text { supplies }\end{array}$ & $\begin{array}{l}\text { Gauze, cotton wool, disposable gloves, Ringers lactate, alcohol swabs, cannula, } \\
\text { plaster, IV tubing set and syringes }\end{array}$ \\
\hline $\begin{array}{l}\text { Hospitalization } \\
\text { costs }\end{array}$ & Food, Water, electricity, Space \\
\hline Stationery & Patient books, pens, checklists, questionnaires, costing forms \\
\hline $\begin{array}{l}\text { Healthcare } \\
\text { personnel }\end{array}$ & $\begin{array}{l}\text { Clinicians time, nurses time, epidemiologists, drivers, helmsman, District Surveillance } \\
\text { Focal Person }\end{array}$ \\
\hline
\end{tabular}

*All the cost categories are direct health care costs taking the public perspective

Table 2: Table showing cost categories

\begin{tabular}{|c|c|c|c|c|c|}
\hline Costs & $\begin{array}{l}\text { Cost } \\
\text { categories }\end{array}$ & Cost component/inputs & How measured & Cost (\$) & $\begin{array}{l}\text { Data } \\
\text { source }\end{array}$ \\
\hline \multirow[t]{3}{*}{ Medical } & \multirow[t]{3}{*}{$\begin{array}{l}\text { Provider } \\
\text { costs }\end{array}$} & Expenditure on treatment & $\begin{array}{l}\text { Costs of } \\
\text { medicines }\end{array}$ & 363 & JMS \\
\hline & & Utilities; electricity and water & cost of units & 100 & Provider \\
\hline & & Transport & $\begin{array}{l}\text { Hire and fuel } \\
\text { cost }\end{array}$ & 5063.3 & Provider \\
\hline \multirow{6}{*}{$\begin{array}{c}\text { Non- } \\
\text { medical }\end{array}$} & \multirow{2}{*}{$\begin{array}{c}\text { Personnel } \\
\text { costs }\end{array}$} & Allowances & Amount paid & 4,574 & Provider \\
\hline & & $\begin{array}{l}\text { salary expended during period of } \\
\text { investigation }\end{array}$ & Amount paid & 5862 & Provider \\
\hline & \multirow{4}{*}{$\begin{array}{l}\text { Overhead } \\
\text { costs }\end{array}$} & Communication & Airtime & 125 & Provider \\
\hline & & Venue hire & cost of venue & 83.3 & Provider \\
\hline & & Stationery & $\begin{array}{l}\text { Cost of } \\
\text { printing }\end{array}$ & 83.3 & Provider \\
\hline & & Loud speaker hire & Cost of hire & 5.6 & Provider \\
\hline \multicolumn{4}{|c|}{ Total } & 16259.5 & \\
\hline
\end{tabular}

\section{Figures}




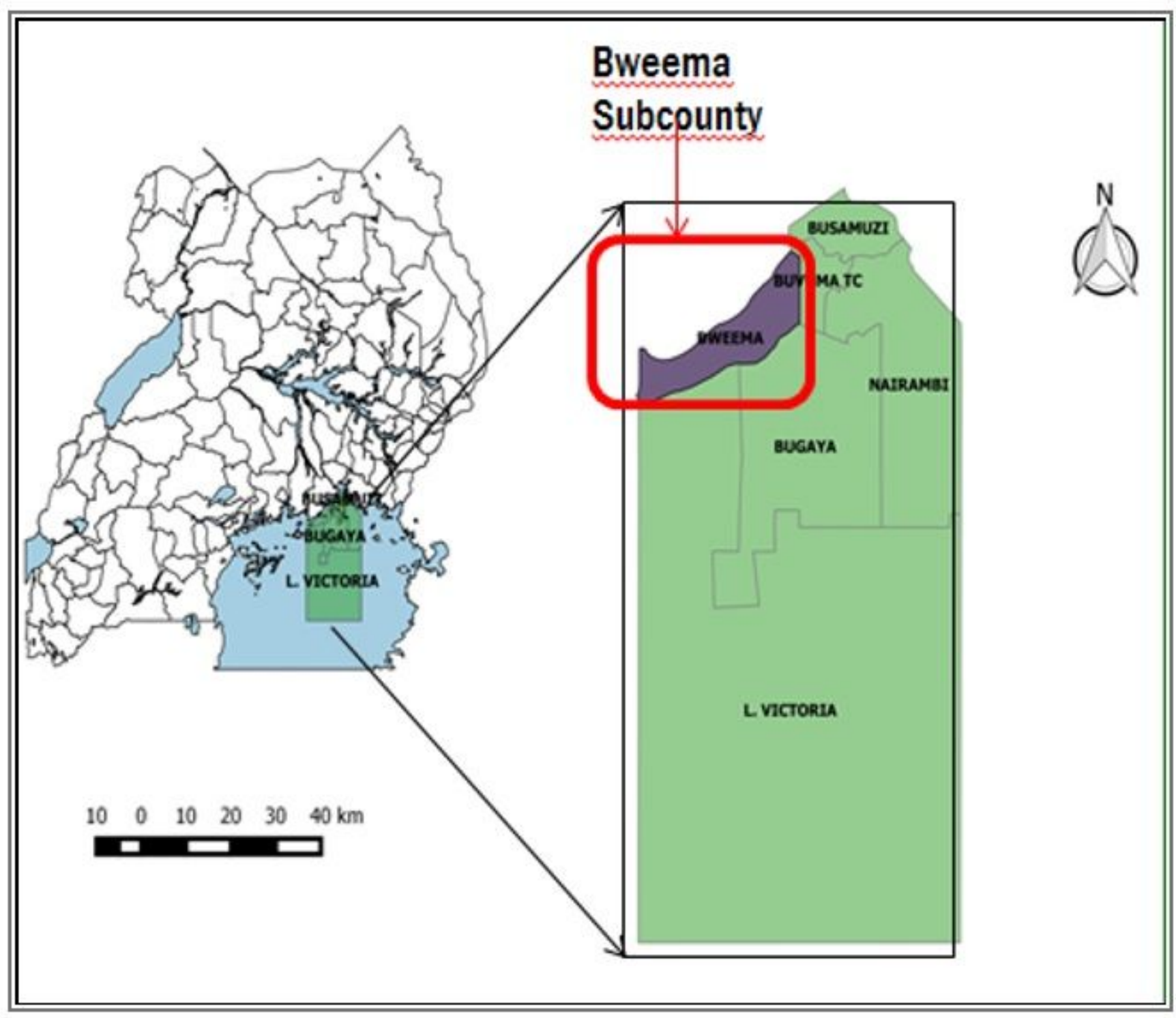

Figure 1

Figure 1: Location of the affected Bweema Subcounty, Buvuma Island, Lake Victoria, 2017

\section{Supplementary Files}

This is a list of supplementary files associated with this preprint. Click to download.

- AdditionalfileCostingchecklist26July.doc 